\title{
The origin and characterization of conformational heterogeneity in adsorbed polymer layers
}

\author{
Jack F Douglas $\dagger$, Hildegard M Schneider $\ddagger$, Peter Frantz $\ddagger$, Robert Lipman \\ and Steve Granick $\ddagger$ \\ $\dagger$ Polymers Division, National Institute of Standards and Technology, Gaithersburg, MD 20899, \\ USA \\ $\ddagger$ Department of Materials Science and Engineering, University of Illinois, Urbana, IL 61801, \\ USA \\ $\S$ Applied and Computational Mathematics Division, National Institute of Standards and \\ Technology, Gaithersburg, MD 20899, USA
}

Received 2 January 1997

\begin{abstract}
The equilibration of polymer conformations tends to be sluggish in polymer layers adsorbed onto highly attractive substrates, so the structure of these layers must be understood in terms of the layer growth process rather than equilibrium theory. Initially adsorbed chains adopt a highly flattened configuration while the chains which arrive later must adapt their configurations to the increasingly limited space available for adsorption. Thus, the chains adsorbed in the late stage of deposition are more tenuously attached to the surface. This type of non-equilibrium growth process is studied for polymethylmethacrylate (PMMA) adsorbed on oxidized silicon where the segmental attraction is strong ( $4 k_{B} T$ /segment) and for polystyrene (PS) adsorbed on oxidized silicon from a carbon tetrachloride solution where the segmental attraction is relatively weak ( $1 k_{B} T /$ segment). Measurements were based on Fourier transform infrared spectroscopy in attenuated total reflection (FTIR-ATR). In both cases, the chains arriving first adsorbed more tightly, became flattened (as measured by the dichroic ratio), and occupied a disproportionately large fraction of the surface. This non-uniform structure persisted indefinitely for the strongly adsorbed PMMA chains, while the PS chains exhibited a gradual evolution, presumably reflecting an equilibration of the adsorbed layer occurring after long times. On the theoretical side, the initial heterogeneity of these adsorbed polymer layers is modelled using a random sequential adsorption (RSA) model where the size of the adsorbing species is allowed to adapt to the surface space available at the time of adsorption. The inhomogeneity in the size of adsorbing species (hemispheres) in this model is similar to the distribution of chain contacts in our measurements on adsorbed polymer layers. Owing to extensive variance around the mean, conformations having the mean number of chain contacts are least probable, which contrasts starkly with expectations based on equilibrium adsorption theory.
\end{abstract}

\section{Introduction}

The morphologies of polymer materials are often governed by kinetic rather than equilibrium parameters (such as energy and temperature) because the complex topologically mediated interactions exhibited by polymer molecules lead to prohibitively long equilibration times and thus to kinetically frozen conformational states. The many internal degrees of freedom of these large molecules allow chemically homogeneous polymer fluids to become geometrically inhomogeneous under non-equilibrium conditions. The nature of these materials consequently depends on the history to which they have been subjected. 
In this paper we study an example of this type of phenomenon: polymers adsorbed from solutions onto solid surfaces. In this field most structural experiments (e.g. neutron scattering and ellipsometry) probe an average surface structure-not the distribution around this average. However, it is probably the common situation that the conformations of adsorbed flexible polymers have a broad, history-dependent conformational distribution when the chains deposit decidedly faster than the surface structure equilibrates. The time scale during which this non-equilibrium structure persists decides the relevance of equilibrium theory for describing the properties of adsorbed polymer layers.

The proposition that conformations of adsorbed flexible polymers have a broad, historydependent distribution has been advanced by experimentalists for many years. Evidence in favour was first advanced during the 1960s and 1970s by Grant et al [1,2]. During the 1980s, Pefferkorn et al proposed the 'hairy carpet' model [3-5], which recognized that chains first arriving at a surface adsorb in relatively flat conformations, while those arriving later become adsorbed in more loosely bound configurations. Even more recently, a similar view arises independently from the Monte Carlo simulations of Zajac and Chakrabarti [6]. Here, building on earlier experimental studies [7-10], we propose unifying principles to characterize non-equilibrium adsorbed polymer layers.

This paper is divided into three parts. First, a simple kinetic model is introduced to model the basic features of non-equilibrium polymer adsorption. We argue that surface structure within a growing polymer layer can be understood based on a consideration of molecules of adjustable size and shape depositing onto the surface. Specifically, we assume that the polymer deposition occurs as a random sequential adsorption of objects (chosen to be hemispherical with a disc-shaped base) where the size of the base discs must be adjusted to fit the available uncovered space where the disc centre lands. This feature of adaptive shape during deposition mimics the restricted space for adsorption due to previously adsorbed chains and the tendency of the chains to form the maximum number of contacts possible when they encounter a strongly adsorbing surface. This model, with a constraint on the upper and lower size of the depositing discs, leads to a distribution of disc areas which is similar to the bound fraction distribution observed in our experiments.

The second section presents experimental evidence supporting the model assumptions of the adaptive random sequential adsorption (RSA) model. The first system considered was a strongly adsorbing polymer, polymethylmethacrylate (PMMA), adsorbed mainly by hydrogen-bonded contacts onto an oxidized silicon ( $\mathrm{Si}$ ) surface from carbon tetrachloride $\left(\mathrm{CCl}_{4}\right)$ or toluene solvents. Expanding on earlier communications [7-9], the surface inhomogeneity was monitored by Fourier transform infrared spectroscopy (FTIR) in attenuated total reflection (ATR) mode. This method allowed us to monitor the distribution of hydrogen bond contacts for the adsorbed chains. The distribution of hydrogen bond contacts to the surface was found to be strongly non-uniform. A U-shaped distribution was observed, most of the chains having either relatively many, or relatively few, hydrogen bonds with the surface. The quantitative characterization of this distinctly non-equilibrium population comprises the first main experimental finding of our study - a key test of the theoretical model.

Finally, we contrast these strong adsorption measurements with measurements where the polymer-surface interaction is weaker and where equilibrium adsorption phenomena might be expected. Polystyrene (PS) is a classical example of weak segment-surface interaction [11] since it has a binding energy of $1 k_{B} T$ per segment for adsorption onto oxidized Si from $\mathrm{CCl}_{4}$ [12]. Expanding on a previous communication [10], our measurements for this system exhibited some features in common with the non-equilibrium adsorption of PMMA, but with the exception that the inhomogeneity of the layer was found to evolve as the surface 
layer 'aged' owing to a higher mobility of the adsorbed PS chains. Whereas the PMMA layer did not exhibit an evolving structure within the limits of our detection, the contrasting PS adsorbed layer gradually became more homogeneous after long times (hours) as the layer presumably evolved towards its final equilibrium state. Nonetheless, the equilibrium time of $9 \mathrm{~h}$ was not sufficient to reach a final equilibration state. Thus, both experimental systems exhibited conformational inhomogeneity associated with the polymer adsorption process.

\section{Theoretical model of non-equilibrium polymer adsorption}

Many experimental data suggest an RSA deposition process when polymer chains adsorb [13-15]. Especially in dilute solution, where the chains intertwine to a limited extent, the chains encounter the surface largely as discrete objects. We can imagine that the sudden transition from the low concentration of the solution, to the high local concentration of the adsorbed layer, suggests that strong interaction between polymers and a solid surface could make the equilibration of conformations very sluggish.

This brings to mind the classical RSA model of particle deposition onto a plane surface, in which particles of fixed size and shape deposit with strict exclusion of overlap between adjoining particles [13]. Once adsorbed, particles cannot diffuse or leave the surface in this model. In the classical RSA model, 'jamming' occurs at an area fraction of about $55 \%$ for idealized rigid circular 'particles' and this has been found useful in characterizing adsorption of certain proteins [15].

How can this model apply to the adsorption of polymers whose shape may adapt to conditions encountered during the deposition process? More specifically, how do we deal with the complication that the many internal chain degrees of freedom allow the chain to reorganize so that adsorption can occur for part of the chain [7]? Flexible polymers can continue to adsorb by adjusting their shape to adsorb at fewer surface sites. This case has been treated independently, especially in the mean-field approximation, in some recent literature [16-20].

We begin with a simple model which abstracts the essential physics by considering particles of fixed volume, but variable shape and cross-sectional size. The classical RSA model of deposition onto a plane surface with exclusion of overlap is enforced as usual, but the disc hemisphere base is required to be the largest size which will fit on the remaining uncovered surface on which the centre of the added hemisphere lies. The centres of the discs are chosen at random on the surface and are allowed only if their centres lie on a previously uncovered region. The special 'adaptive' nature of the deposition process means that full coverage is ultimately obtained when there is no lower cut-off size; thus, the jamming limit approaches unity as the cut-off is made smaller, which contrasts strongly with the classical RSA model. The adaptive modification is a natural generalization of the RSA model to describe the deposition of 'soft' objects such as polymers, vesicles, and flexible proteins whose shape can adjust to the changing molecular environment of the surface during the deposition process. The seemingly simple 'adaptive' criterion in the RSA model profoundly changes the character of the deposition geometry.

In figure 1 we show a realization of the adaptive random sequential adsorption (ARSA) model which exhibits inhomogeneity on multiple scales. This contrasts sharply with the rather uniform surface coverage in the ordinary RSA model (see figure 2 and [21]), which results from excluded volume interactions. One observes a population of objects with large cross-sectional area, separated by numerous objects with smaller cross-sectional area. Indeed, if one enlarges any portion of the deposited layer in figure 1 (see figure 3 ), the 


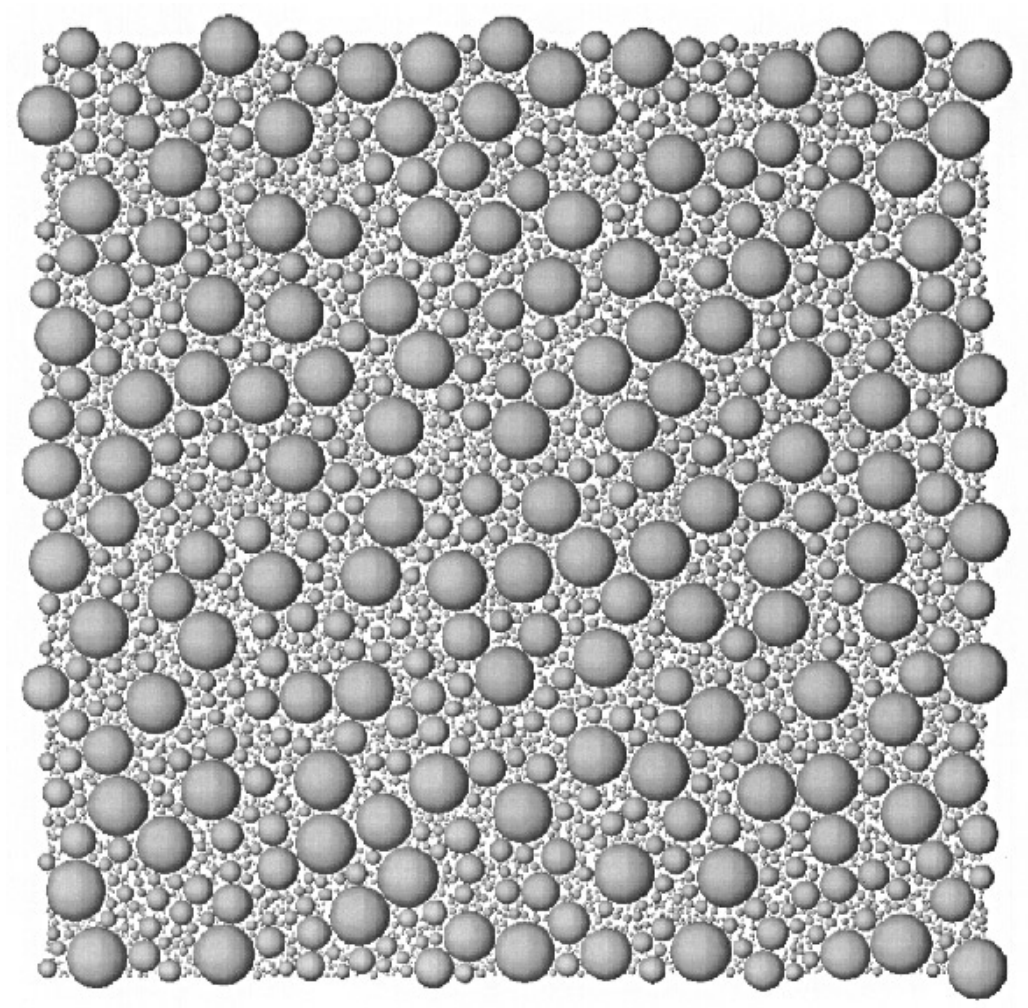

Figure 1. A computer realization of the ARSA model. The classical RSA model of deposition onto a plane surface with exclusion of overlap is enforced as usual (once adsorbed, particles cannot diffuse or leave the surface), but the disc forming the hemisphere base size is required to be the largest size which will fit on the remaining uncovered surface on which the centre of the added hemisphere lies. The special 'adaptive' nature of the deposition process means that full coverage is ultimately obtained in the limit of vanishing lower cut-off scale. The preparation of figure 1 corresponds to $\lambda=0.05$. The ratio of the upper cut-off size to the size of the region on which the discs are deposited is another relevant scale in this deposition process and in figure 1 the length of the square region is equal to 30 times the radius of the largest disc base.

resulting 'bubble-pattern' appears nearly the same as the original. This is the hallmark of a 'fractal' pattern.

Figure 3 shows a representative enlargement of a square section of figure 1. The pattern becomes 'fractal', i.e. has no characteristic length scale, in the limit where so many hemispheres have been added that the uncovered surface has no measurable area. As mentioned above, this corresponds to the limit of a vanishing lower cut-off and no bounded upper cut-off. The pattern of the hemisphere bases (discs) on the plane surface in figure 1 clearly resembles the well known Appolonian packing which has been known since antiquity (200 B.C.). Accurate estimates of the fractal dimension of the uncovered part of the plane are known for the classical Appolonian packing: $d_{f}=1.305684$ [22]. Recent estimates of the randomized analogue of Appolonian packing corresponding to our ARSA model with the circular boundary give a somewhat larger value of the fractal dimension, $d_{f} \approx 1.62-1.64$ [23]. After the present study was undertaken, the significance of Appolonian packing in surface deposition problems was also recognized in other studies $[16,19]$. It is notable that 


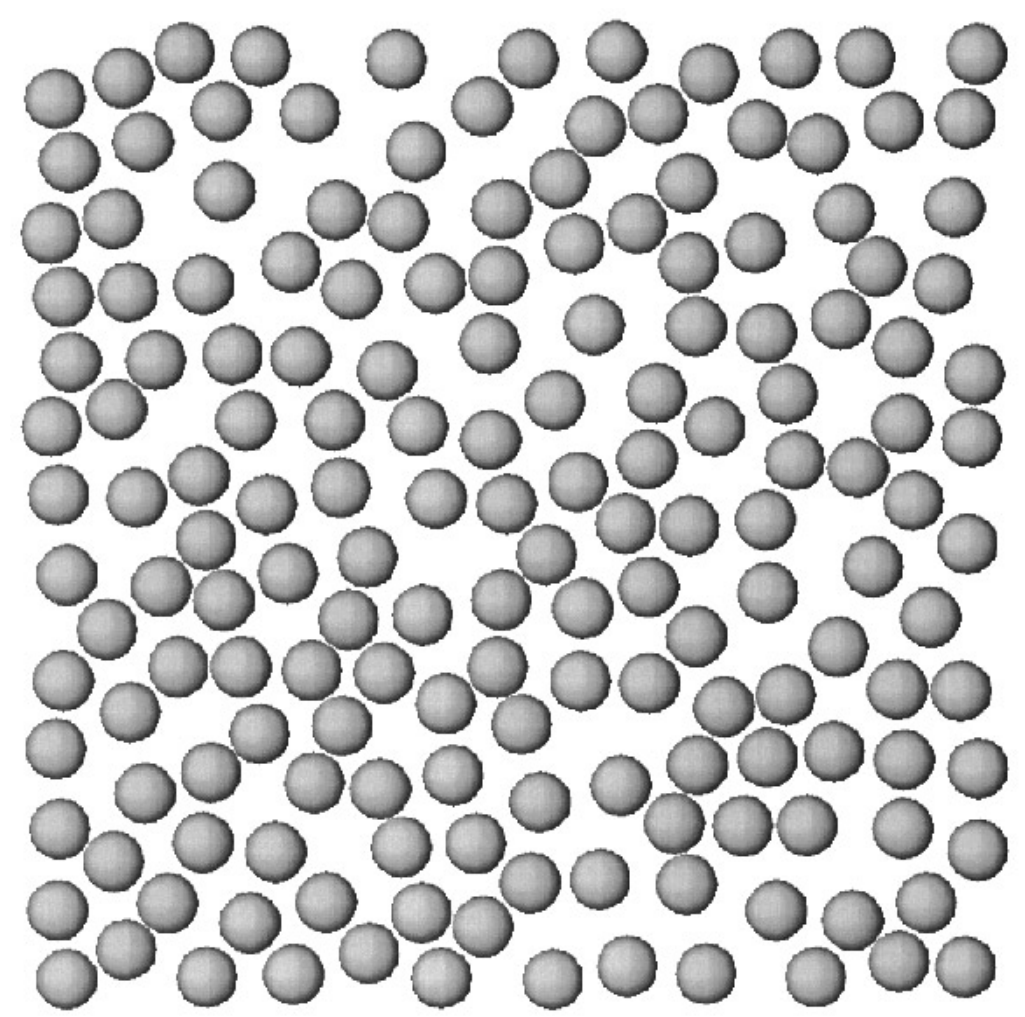

Figure 2. An illustration of jamming coverage for the classical RSA model of particle deposition onto a plane surface without adaptation: particles of fixed size and shape deposit with strict exclusion of overlap between adjoining particles. In this model, 'jamming' occurs at an area fraction of about $55 \%$ for rigid particles. In the figure, the number 169 indicates that jamming occurred after deposition of 169 hemispheres. Jamming at relatively low surface coverage contrasts with the ARSA model illustrated in figures 1, 3, and 4.

this statistical model of surface structure has nothing to do with equilibrium theory, which is the traditional way of modelling adsorbed polymers.

In the real polymer deposition problem, an upper cut-off limit is also set by the maximum area to which a chain of given length can spread to occupy during the adsorption process. A lower cut-off limit is set by the minimum number of bonds between the polymer and the surface that is required to produce firm surface attachment. In the ARSA model, these constraints have analogues in the maximum and minimum disc base sizes, respectively, for the deposited hemispheres. The existence of upper and lower limit disc base sizes inhibits the development of a fully fractal pattern and causes the coverage to 'jam' at a value which increasingly approaches unit coverage as the ratio $(\lambda)$ of the smallest disc radius to the largest disc radius decreases. The preparation of figures 1 and 3 corresponds to $\lambda=0.05$. The ratio of the upper cut-off size to the size of the region on which the discs are deposited is another relevant scale in this deposition process and in figure 1 the length of the square region is equal to 30 times the radius of the largest disc base.

Upon comparison with experiments (see below), we observe that the area distribution in the ARSA model with an upper and lower cut-off is quite similar to the experimentally derived measurements of the bound fraction distribution, where the bound fraction in the 


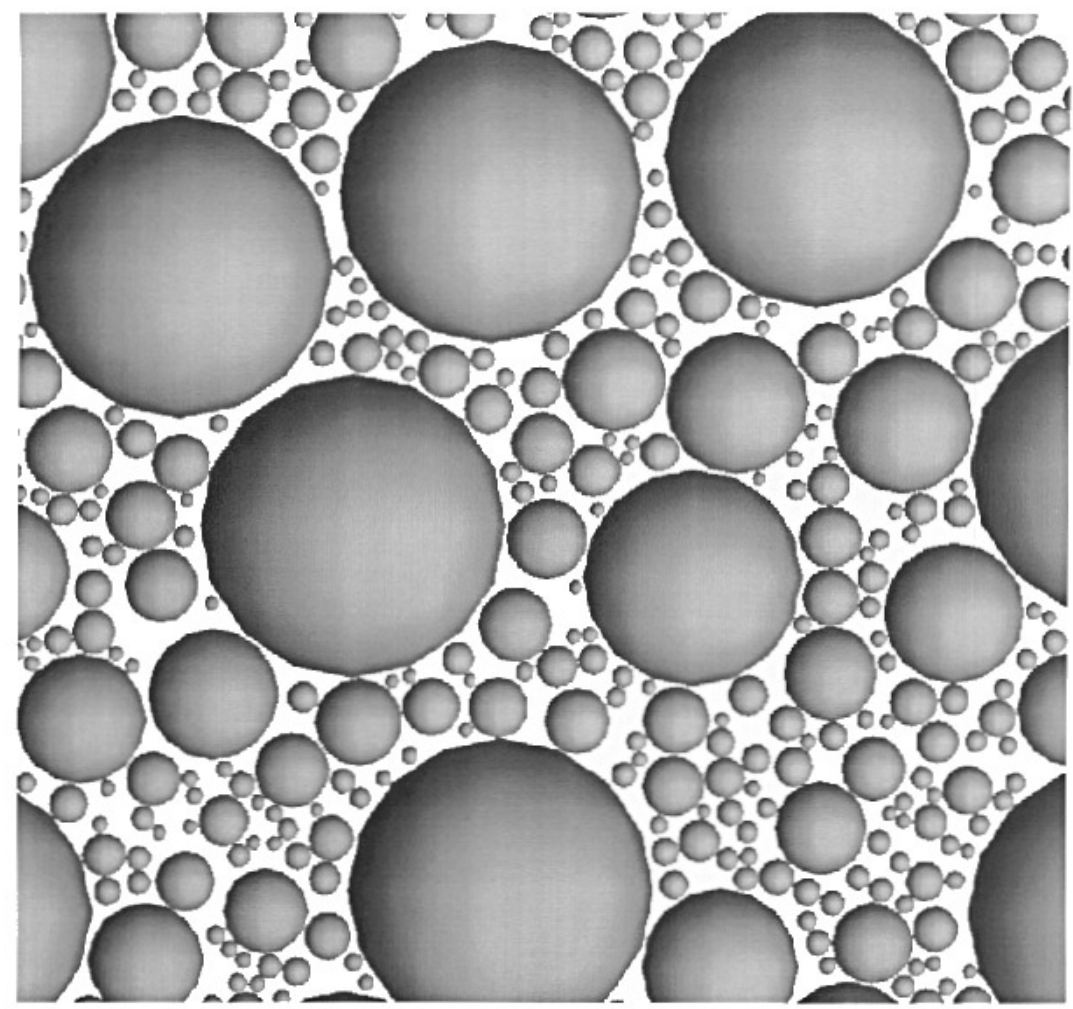

Figure 3. A blow-up of a representative section of figure 1. Because the model has no characteristic length scale, the adsorbate develops a 'fractal' structure in the limit where so many hemispheres have been added that the uncovered surface has no measurable area. The fractal limit is strictly only achieved when the lower cut-off of the object size is vanishingly small, while the visualization requires a finite but small lower cut-off. The pattern of the hemisphere base discs on the plane surface resembles the well known Appolonian packing which has been known since antiquity.

polymer adsorption problem (see below) should be compared to the number fraction of the deposited discs.

Figure 4 shows a typical U-shaped distribution of disc sizes corresponding to disc size parameters indicated above for figure 1. The data in the top panel are for an early stage of the deposition where about 500 hemispheres have been deposited, while the data in the bottom panel show the relatively late stage where about 6000 hemispheres occupy the surface. For the late-stage coverage, the distribution has become skewed by the increasing number of small hemispheres. The ideal 'fractal' regime associated with fully developed coverage and the limit $\lambda \rightarrow 0^{+}$is approached by this data. Increasing the lower cut-off size $(\lambda)$ causes jamming at an earlier stage of deposition so the distribution becomes more U-shaped, closer to the pattern in figure 4, top panel.

Another refinement of the ARSA model which could provide further insights into nonequilibrium polymer adsorption is to require the volume of the hemisphere 'droplet' to remain invariant by replacing the hemispheres by spherically capped circular cylinders oriented normal to the surface, where the volume of the cylinders is forced to have the same volume as the largest hemisphere in the former calculations. This model is natural 

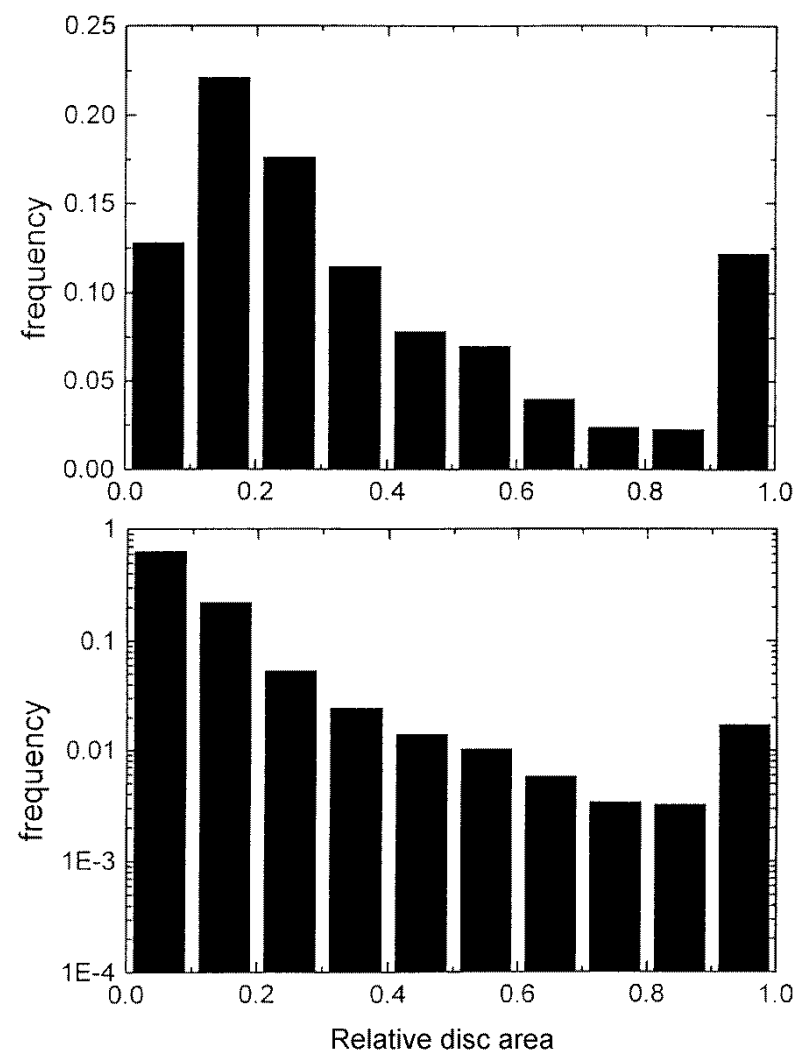

Figure 4. Adsorption area distribution in the adaptive random sequential adsorption (ARSA) model. The histogram is formed by sorting the radii of discs (for calculations of the type illustrated in figure 1) into ten bins. The largest discs are normalized to have unit radius and the sizes of the smaller discs are measured relative to this reference scale. The ratio $(\lambda)$ of the smallest disc radius to the largest disc radius was $\lambda=0.05$. Top panel, early stage of deposition, in which 519 particles have deposited. The ordinate axis is linear. Bottom panel, late stage of deposition, in which 5812 particles have deposited. The ordinate axis is logarithmic. The ratio of the upper cut-off size to the size of the region on which the discs are deposited is another relevant scale in this deposition process; the length of the square region is equal to 30 times the radius of the largest disc base.

if we idealize the adsorbed polymer chains as being like deformable droplets with constant volume. An example of this model is shown in figure 5, which shows a view from the side. The 'matted carpet' surface layer in this figure shows appreciable fluctuations in height, which naturally accompany the fluctuations in the bound area within the plane. The structure of this layer normal to the surface evidently becomes 'fractal' in the limit of a vanishing lower cut-off on the disc radius. Such large fluctuation in the local thickness of the adsorbed polymer layer would probably be reflected indirectly in hydrodynamic thickness measurements [7].

Experimental evidence of layer 'fuzziness'. in non-equilibrium adsorption comes from recent measurements by Auroy and Auvray of the adsorption of polydimethylsiloxane (PDMS) on porous silicon. Small-angle neutron scattering shows the polymer layer to be diffuse and these layers have been termed 'pseudo-brushes' [24]. 


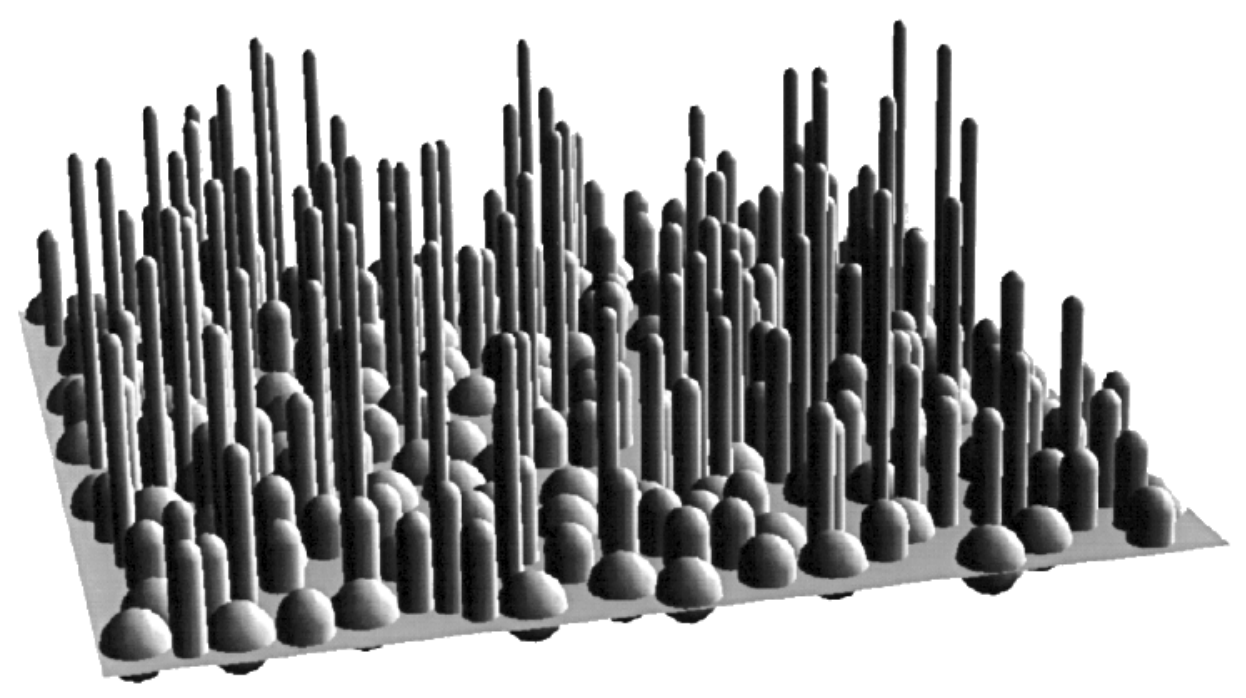

Figure 5. A computer realization of the ARSA model in which we idealize the adsorbed polymer chains as deformable droplets with constant volume with cylindrical shape. The figure illustrates the resulting 'matted carpet' surface, in which appreciable fluctuation in the height of the surface layer naturally accompanies the fluctuations in the bound area within the plane. The computer realization is a side view with parameters the same as in figures 1 and 3.

\section{Experiments with PMMA (strongly adsorbing) polymer}

\subsection{Experimental approach}

Characteristics of the atactic linear PMMA chains are given in table 1. The measurement temperature was $30.0^{\circ} \mathrm{C}$. The solid absorbing surface was oxidized silicon. The methods to clean $\mathrm{Si}$ and to grow a surface oxide layer in a controlled manner have been described previously [25]. Also described previously are the methods used to make measurements with polarized radiation [26] and to calibrate the infrared absorbance in terms of mass adsorbed [26]. The ATR experiments were performed with an FTIR spectrometer equipped with a broadband mercury cadmium telluride detector.

In this system, PMMA adsorbs mainly by hydrogen bonding and shows no adsorption isotope effect [27]. The net segment-surface interaction energy was determined to be $4 k_{B} T$ [28] ( $k_{B} T$ denotes thermal energy units) using the method pioneered by Cohen Stuart and coworkers [11]. Carbon tetrachloride was the usual solvent, except for control experiments discussed below, because it is so transparent at infrared wavelengths.

Each segment of a PMMA molecule contains a carbonyl group. The bound fraction is defined as the fraction of carbonyl groups of adsorbed chains that are hydrogen bonded to the surface:

$$
p=\Gamma_{b} / \Gamma_{f}
$$

where $\Gamma_{b}$ is the calibrated surface coverage of chains bound to the surface [9], and $\Gamma_{f}$ is the calibrated surface coverage of carbonyl groups. The bound fraction gives information about the relative 'flatness' of the adsorbed layer. 
Table 1. Characteristics of the PMMA and PS chains (suppliers' data). According to ISO 31-8, the term 'molecular weight' has been replaced by 'relative molecular mass' with the symbol $M_{r}$. The older conventional notation is used in the present work.

\begin{tabular}{|c|c|c|c|c|}
\hline Sample & $M_{w}^{\mathrm{a}}$ & $M_{w} / M_{n}$ & Isotope & Source \\
\hline PMMA & 7700 & 1.15 & protio & $\mathrm{PL}^{\mathrm{b}}$ \\
\hline PMMA & 64000 & $<1.09$ & protio & PL \\
\hline PMMA & 90000 & - & deuterio & $\mathrm{PSS}^{\mathrm{c}}$ \\
\hline PMMA & 107000 & $<1.10$ & protio & PL \\
\hline PMMA & 1300000 & 1.06 & protio & PL \\
\hline PS & 85000 & 1.05 & deuterio & PL \\
\hline PS & 87000 & 1.05 & deuterio & PL \\
\hline PS & 550000 & 1.05 & deuterio & PL \\
\hline PS & 575000 & 1.06 & protio & $\mathrm{PC}^{\mathrm{d}}$ \\
\hline PS & 760000 & 1.06 & protio & $\mathrm{TS}^{\mathrm{e}}$ \\
\hline
\end{tabular}

${ }^{a}$ Atactic chains.

b Polymer Laboratories.

${ }^{c}$ Polymer Standards Service.

${ }^{\mathrm{d}}$ Pressure Chemical.

e Toyo Soda.

\subsection{Kinetically frozen surface conformations}

The ARSA model described in the theoretical section presupposes a wide separation in time scales: the surface structure of adsorbed chains should adapt to the available surface space more quickly than the time scale of subsequent adsorption, and thereafter remain static. How to test this experimentally?

The first evidence that adsorbed chain structure was kinetically frozen in historydependent conformations came from experiments in which PMMA was allowed to deposit uninterruptedly onto the initially bare silicon oxide surface. In figure 6 , one sees that the deposition was rapid at very low surface coverage, consistent with diffusion-limited adsorption [29], but soon slowed. In parallel experiments, the solid surface was exposed, at two points of incomplete surface coverage, to solutions of isotopically labelled molecules of matched molecular weight. As mentioned previously, the system shows no adsorption isotope effect [27]. No desorption of the former polymer accompanied subsequent adsorption by the labelled sample.

Further evidence of a kinetically frozen structure came from inspection of the infrared dichroism. The dichroic ratio was defined as the ratio of absorptivity perpendicular to the surface and in one direction parallel to it, such that a dichroic ratio of unity would indicate isotropy. Figure 6, lower panel, shows, for the same experiments as shown in the upper panel, the dichroic ratio of the asymmetric methyl stretch of adsorbed PMMA chains, $D_{\mathrm{CH}_{3}}$. The data show anisotropy, a preference for vibrations in the plane of the surface, i.e. $D_{\mathrm{CH}_{3}}<1$. This anisotropy was most intense when the surface coverage was lowest. The experiments with isotopic labelling showed that the mean segmental orientation of the earliest-adsorbed chains remained fixed in a state far more flattened than the average for the entire layer-and also showed no history dependence.

The time dependence of the bound fraction is shown in figure 7. The bound fraction of the layer was largest at the earliest times (lowest surface coverage) and then decreased. The experiments in figure 6 showed that the chain conformations were not equilibrated, however. Therefore, as adsorption proceeded to completion, initially absorbed chains having many surface contacts were buried under a ponderous cover of chains having relatively few surface 

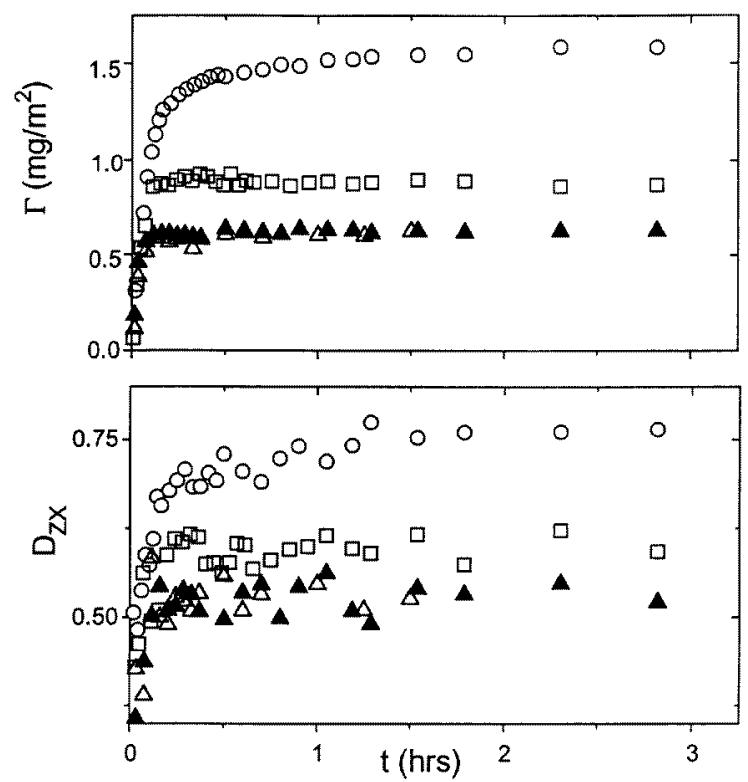

Figure 6. Adsorption of PMMA $\left(M_{w}=107000 \mathrm{~g} \mathrm{~mol}^{-1}\right.$ at a concentration of $0.01 \mathrm{mg} \mathrm{ml}^{-1}$ in $\mathrm{CCl}_{4}$ ) plotted against elapsed time. Top panel, mass adsorbed per area $(\Gamma)$ during uninterrupted adsorption (circles). Alternatively, the solution was replaced by isotopically labelled PMMA $\left(M_{w}=90000\right)$ at the same solution concentration (open symbols) or at 100 times larger concentration (filled symbols) after 4 min (triangles) or after 8 min (squares). The $\Gamma$ of polymer D was unaffected. Bottom panel, the dichroic ratio $\left(D_{C_{3}}\right)$ of the asymmetric $\mathrm{CH}_{3}$ vibration for the experiments indicated in the top panel. A dichroic ratio of unity would indicate isotropy. The symbols are same as for the top panel.

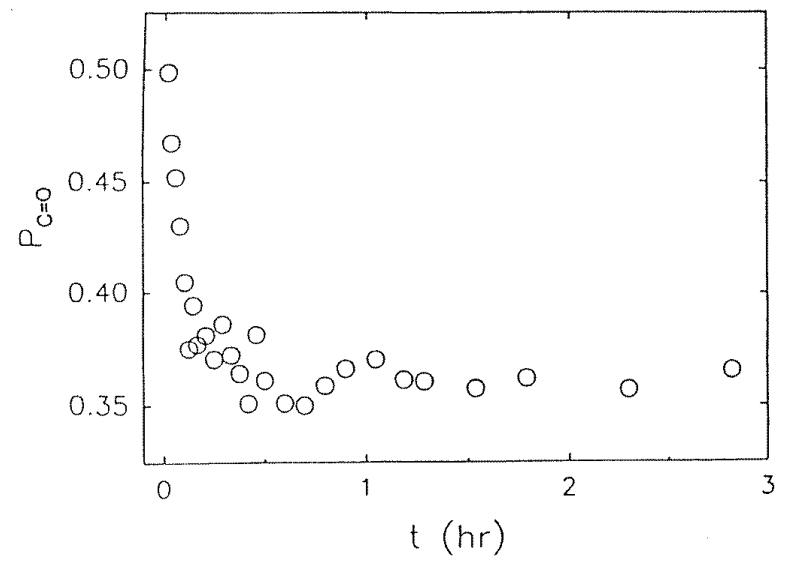

Figure 7. The bound fraction, $p_{C O}$ (hydrogen-bonded mass divided by total adsorbed mass) for uninterrupted adsorption (data of figure 6). This average quantity is masking a distribution of structures (see discussion in text).

contacts [7]. This disparity in the contacts makes the initially adsorbed chains more highly flattened than the chains adsorbing at later times, as indicated by the dichroic ratio. 


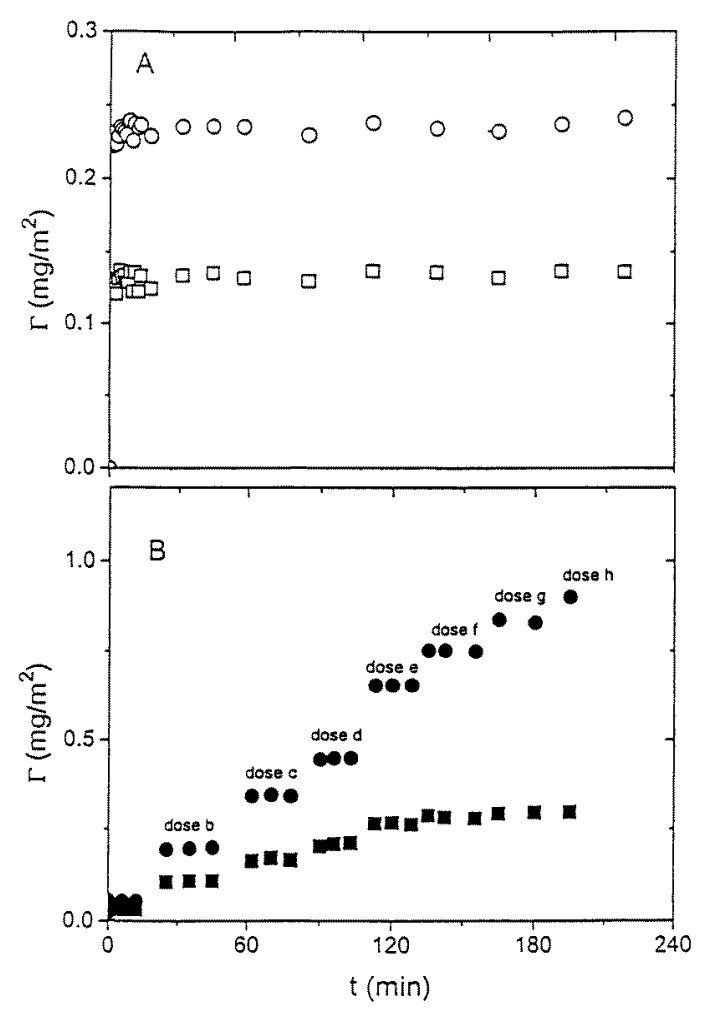

Figure 8. Mass adsorbed plotted against elapsed time for surfaces 'starved' of PMMA $\left(M_{w}=7700 \mathrm{~g} \mathrm{~mol}^{-1}\right.$ ). Two experiments are shown. (A) Adsorption (from $0.01 \mathrm{mg} \mathrm{ml}^{-1}$ in $\mathrm{CCl}_{4}$ ) to a point of incomplete surface coverage followed by replacement of the solution with pure $\mathrm{CCl}_{4}$ solvent. Total mass adsorbed (circles) and bound mass (squares), hydrogen bonded to the surface, were constant with elapsed time. (B) At periodic intervals the adsorbed layer was re-exposed to the same solution of PMMA $\left(M_{w}=107000 \mathrm{~g} \mathrm{~mol}^{-1}\right)$ for 5-10 s. Total mass adsorbed (filled circles) and bound mass (filled squares), hydrogen bonded to the surface, were constant with elapsed time.

\subsection{Rapid surface spreading in response to the available surface area}

The next series of experiments showed that the rate of spreading was more rapid than the rate of adsorption; in fact, so rapid that it was accomplished before the first data could be acquired. Our initial expectation had been somewhat different: we reasoned that if the surface were 'starved' of polymer by depositing a small amount, too small to saturate the surface, this would provide a driving force for the chains to 'spread out', i.e. to continue to flatten in order to maximize the number of segment-surface contacts. Experiments showed that this process was completed rapidly, however. Figure 8(A) shows this binding measurement: the bound fraction reached a constant level more rapidly than data could be acquired, and subsequently did not change even when one monitored the bound fraction for $4 \mathrm{~h}$. Samples having other molecular weights and concentrations led to similar kinetics.

By this method of 'dosing' the solid surface with small increments of surface coverage, the incremental mass of bound segments, attributable to incremental aliquots of adsorbed chains, was determined. Figure 8(B) shows an example. As in figure 8(A), both the total mass adsorbed and the hydrogen-bonded bound mass adsorbed were stable, indicating that 


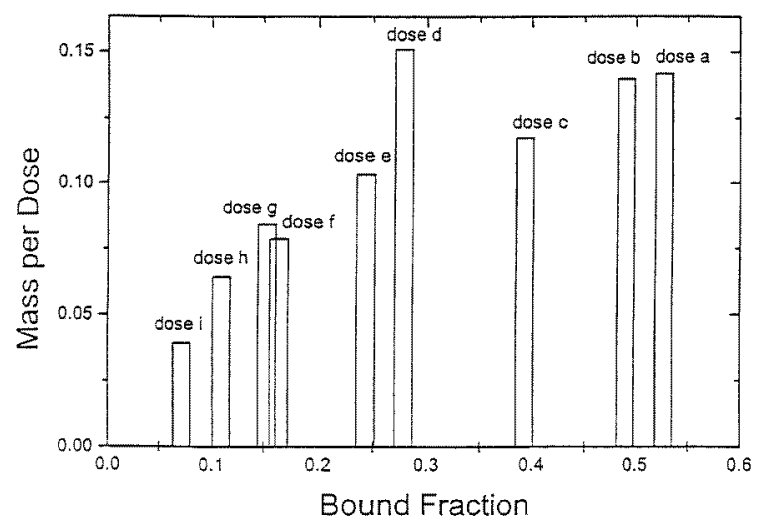

Figure 9. The mass per dose (in figure 8) plotted against its average bound fraction. The doses were exposed to the previously bare surface in alphabetical order (a, b, c, and so forth). The data refer to PMMA $\left(M_{w}=90000 \mathrm{~g} \mathrm{~mol}^{-1}, 0.01 \mathrm{mg} \mathrm{ml}^{-1}\right.$ in $\left.\mathrm{CCl}_{4}\right)$.

the surface structure was kinetically frozen.

For each dose of mass adsorbed in figure 8(B), the average bound fraction was determined. In figure 9, the mass of each dose is plotted against its average bound fraction. One observes that the bound fraction was largest for the doses that were deposited at the lowest surface coverage (e.g. doses a and b). The bound fraction was of the order of five times less for the doses that were deposited at the highest surface coverage (e.g. doses $h$ and i).

\subsection{Quantification of the bound fraction distribution}

Figure 10 shows a one-to-one correspondence between $\Gamma_{b}$ and total surface excess, regardless of molecular weight or adsorption technique. Some of the data refer to uninterrupted adsorption as illustrated in figure 6. Other data refer to dosed adsorption as illustrated in figures 8 and 9. Data from a control experiment in which the solvent was changed from $\mathrm{CCl}_{4}$ (a near-theta solvent) to toluene (good solvent) are also indicated and found to be consistent with the $\mathrm{CCl}_{4}$ measurements. Other control experiments, in which the polymer was desorbed by adding polar displacer molecules, verified the absence of chemisorption [25]. These measurements show that the strong polymer-surface interactions dominated over the effect of solvent quality.

How do we characterize and model these heterogeneous surface structures? The key point allowing this was the observation, validated by the experiments just discussed, that the conformations were kinetically frozen. The brief, the analysis was as follows. From the change of $\Gamma_{b}$ that resulted from a stipulated incremental mass of chains adsorbed $\left(\Gamma_{\text {total }}\right)$, the quantity $p^{\prime}$ (the bound fraction of these chains) was determined by subtracting successive measurements of bound mass. From these subtractions the histogram of mass adsorbed, attributable to sub-populations of chains with different bound fractions $p^{\prime}$, was inferred. Details of the calculation are described elsewhere [7, 8].

The resulting histograms of bound fraction $p^{\prime}$ are plotted in figure 11. Figure 11(A) shows the histogram inferred from the uninterrupted adsorption whose raw data are shown in figures 6 and 7. Figure 11(B) shows the histogram inferred from the numerous dosing experiments summarized in figure 9 . 


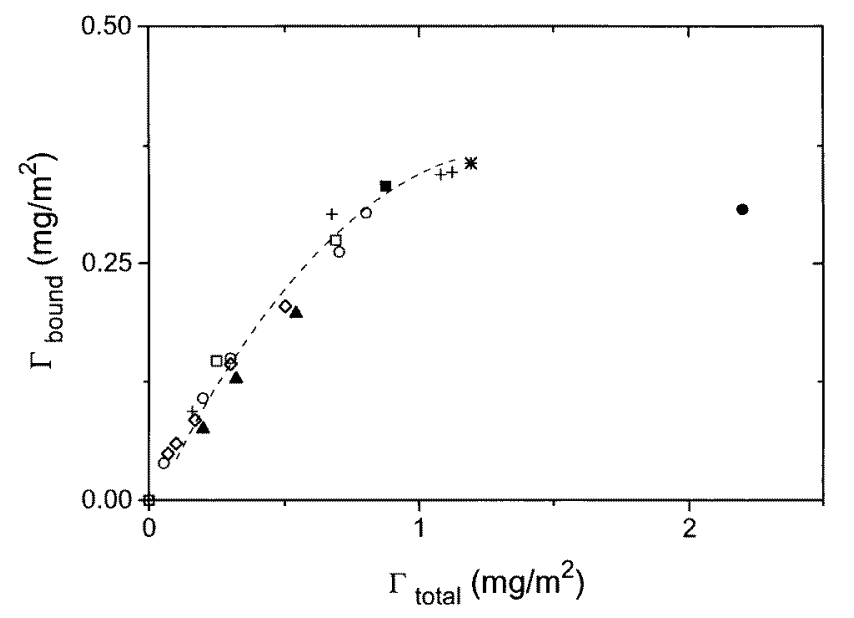

Figure 10. Bound mass (hydrogen bonded to the surface) plotted against total mass adsorbed. Dashed line, uninterrupted adsorption, from data in figure 6. Dosage from $0.01 \mathrm{mg} \mathrm{ml}^{-1}$ in $\mathrm{CCl}_{4}$ except for the case of toluene (specified below): open circles, PMMA, $M_{w}=90000 \mathrm{~g} \mathrm{~mol}^{-1}$; diamonds, PMMA, $M_{w}=107000 \mathrm{~g} \mathrm{~mol}^{-1}$; filled squares, PMMA, $M_{w}=64000 \mathrm{~g} \mathrm{~mol}^{-1}$; open squares, PMMA, $M_{w}=7700 \mathrm{~g} \mathrm{~mol}^{-1}$. Other dosage from $\mathrm{CCl}_{4}$ : star, PMMA, $M_{w}=64000 \mathrm{~g} \mathrm{~mol}^{-1}$ at $0.08 \mathrm{mg} \mathrm{ml}^{-1}$. A control experiment was performed from toluene solvent: plus signs, PMMA, $M_{w}=107000 \mathrm{~g} \mathrm{~mol}^{-1}$ at $0.01 \mathrm{mg} \mathrm{ml}^{-1}$ in toluene. Another control experiment involved adsorption from a semidilute solution: filled circle, PMMA, $M_{w}=107000 \mathrm{~g} \mathrm{~mol}^{-1}$.

The distributions divide into two primary populations. One population was comprised of those chains that met a bare surface and sank into highly flattened configurations, so strongly adsorbed that their structure was effectively frozen in time. The second population was comprised of those chains that, meeting a nearly saturated surface, became attached more tenuously. The numerical values of the calculated end points of the distribution fluctuated somewhat according to the set of raw data used, and also according to choices of how to fit the residual scatter in the raw data, but the finding of a U-shaped distribution is certain.

\subsection{Adsorption onto previously adsorbed poly(n-butylmethacrylate)}

If the surface silanol groups were occupied by prior adsorption by other polymer chains, would newly arrived polymer chains be capable of adsorbing? To address this problem, a two-stage experiment was carried out. First, the bare surface was exposed to poly $(n-$ butylmethacrylate) (PBMA). (The PBMA was purchased from Polymer Laboratories. Its weight-average molecular weight was $M_{w}=103700 \mathrm{~g} \mathrm{~mol}^{-1}$ and the ratio to the numberaverage molecular weight was $M_{w} / M_{n}=1.05$.) Sufficient time was left to ensure saturation of the mass adsorbed. Next the solution of PBMA was replaced by a solution of PMMA at the same concentration. As shown in figure 12, the total mass adsorbed increased by over $50 \%$ with minimal $(5 \%)$ change in the bound mass, indicating that the newly arrived PMMA chains were attached very tenuously, i.e. with a bound fraction $<0.05$.

In this experiment the distribution of populations was apportioned between the two chemical species: PBMA (relatively flattened at the surface) and PMMA adsorbed onto the PBMA (adsorbed tenuously). 

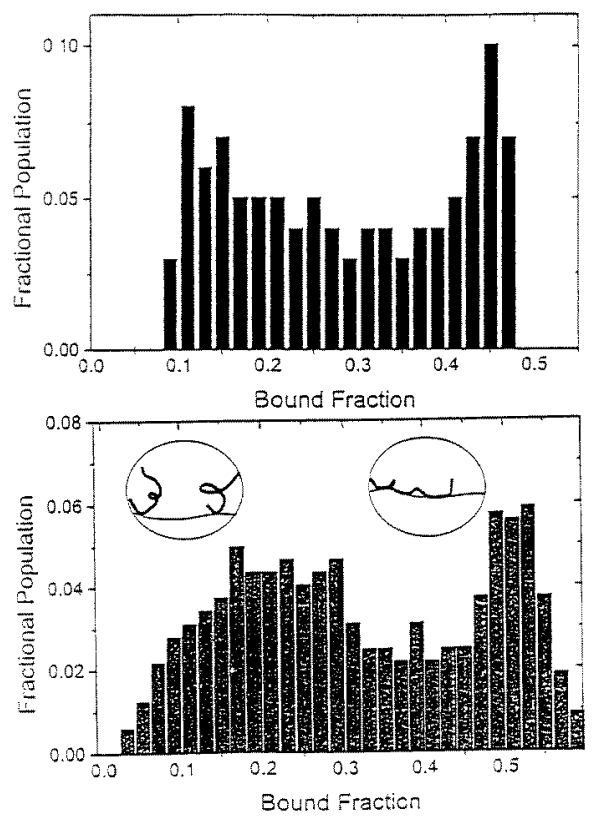

Figure 11. A histogram of bound fraction determined, for bound fractions separated by $\Delta p^{\prime}=0.02$, from data in the above figures. To avoid propagation of errors as incremental differences became small, the histograms were evaluated at a surface excess of $1.2 \mathrm{mg} \mathrm{m}^{-2}$. The inset shows a schematic illustration of chains with large bound fraction and small bound fraction. (A) Bound mass against total mass from uninterrupted adsorption shown in figure 6 . (B) Bound mass against total mass from dosing experiments shown in figures 8 and 9.

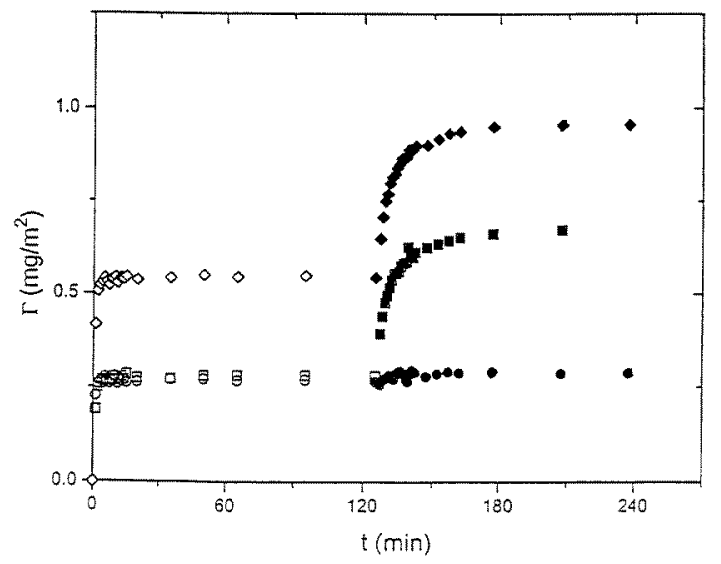

Figure 12. Mass adsorbed plotted against elapsed time for PMMA adsorbed onto a previously adsorbed layer of PBMA $\left(M_{w}=102700\right.$ and $\left.M_{w} / M_{n}=1.05\right)$ : open symbols, PBMA; filled symbols, PMMA; diamonds, total mass adsorbed; circles, bound mass adsorbed; squares, free mass adsorbed.

\subsection{Tests of surface homogeneity}

One might argue that surface chemical or other inhomogeneities could be an additional cause of inhomogeneous adsorption. Many control experiments were performed to test this 
possibility. Here we summarize these, and why we do not believe that this had significant influence on our measurements.

Several pieces of evidence indicate the lack of appreciable inhomogeneity. While some common methods of surface preparation do produce a large amount of demonstrable inhomogeneity [25], the method to produce a homogeneous layer of oxide on a silicon crystal was found to be effective in producing a homogeneous surface as determined by several experimental probes [25]. The most direct evidence came from displacement measurements by adding a small-molecular polar co-solvent (methylene dichloride in the present experiments). Above a certain critical displacer volume fraction, termed the 'critical volume fraction', the polymer was completely displaced. An elegant technique, developed by Cohen Stuart et al, provides a relationship between this point and the segmental adsorption energy [11]. Displacement experiments performed using silicon oxides prepared by exposure to oxygen plasma [25] showed the same critical volume fraction regardless of aging time, $30 \mathrm{~min}$ or $24 \mathrm{~h}$, though alternative methods of surface preparation did not. We conclude that the average strength of the segment-surface interaction remained constant.

Other evidence arises from the observation that the magnitude of the aging time constant depends markedly on the composition of the solution though the surface preparation is kept constant. Studies showed that the aging time constant changes with polymer concentration in solution and increases strongly with increasing molecular weight of the polymer [30,31].

\section{Experiments with PS (weakly adsorbing) polymer}

A limitation of the studies with PMMA was that PMMA adsorbed so strongly ( $\sim 4$ $k_{B} T /$ segment) that the generality of the experimental finding of a non-homogeneous polymer layer structure was uncertain. A weakly adsorbing system was next considered to see what influence the tendency towards equilibration would have on the polymer layer structure. Atactic PS is perhaps the most widely studied model system in previous adsorption experiments involving polymers in non-polar solvents. This polymer seemed appropriate for the present study of weak adsorption since the adsorption of PS has been considered a benchmark for testing the equilibrium theory of polymer adsorption [11]. As noted above, the sticking energy for PS adsorbed from carbon tetrachloride is $1 k_{B} T /$ segment [12], which is relatively weak. Though adsorbed PS shows no direct infrared measures of adsorbed structure (neither chain flattening nor bound fraction can be measured for this polymer by infrared spectroscopy), the displacement of adsorbed chains in response to a given driving force turned out to provide an indirect probe of adsorption homogeneity. Experimental procedures and methods were the same as for PMMA, except that the temperature was $25.0{ }^{\circ} \mathrm{C}$ for the experiments with PS. A more detailed account of the experiments summarized below is presented elsewhere [10].

The starting point was that adsorbed chains could not be displaced completely by their isotopic counterpart unless the solution concentration was exceptionally high. An example of the raw data is shown in figure 13. The mass adsorbed is plotted as a function of elapsed time. First, protio-PS (H-PS) was allowed to adsorb onto the initially bare surface, allowed to equilibrate for some time $(8 \mathrm{~h})$, and finally the H-PS in solution was replaced by deuterated PS (D-PS) of matched molecular weight. Conformational heterogeneity was suggested by the observation that a fraction of the originally adsorbed layer failed to be displaced, in spite of equilibration times of hours and weak segmental sticking energy of the PS monomeric unit, when exposed to solutions comprised exclusively of the isotopically labelled counterpart.

It was desirable to vary the displacement driving force in increments smaller than could 


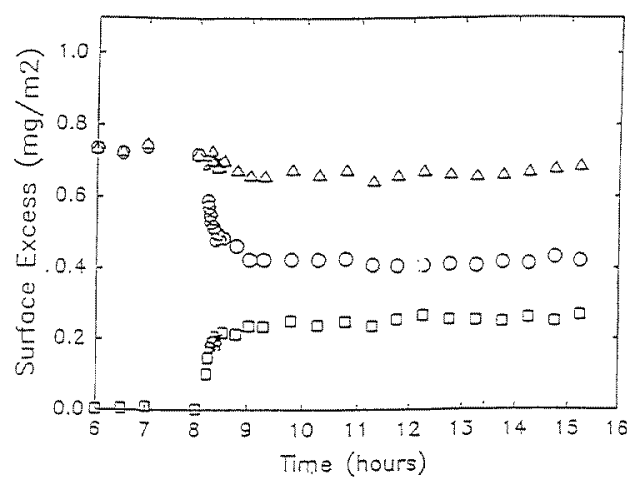

Figure 13. Surface excess of protio- and deuterio-PS plotted versus elapsed time. First H-PS $\left(M_{w}=575000,1 \mathrm{mg} \mathrm{ml}^{-1}\right.$ ) was allowed to adsorb for $8 \mathrm{~h}$, then H-PS solution was replaced by D-PS solution $\left(M_{w}=550000,1 \mathrm{mg} \mathrm{ml}^{-1}\right)$ and the time-dependent changes of mass adsorbed were monitored. Triangles, total mass adsorbed; circles, H-PS; squares, D-PS [10].

be achieved by changing the molecular weight. Therefore, the concentration of the displacer solution was varied (while holding the molecular weight constant). The molecular weight of the initially adsorbed (deuterio) chains was $87000 \mathrm{~g} \mathrm{~mol}^{-1}$; this deuterio sample was exposed first to the surface because this procedure consumed less of these costly samples. The molecular weight of the displacer (protio) chains was $575000 \mathrm{~g} \mathrm{~mol}^{-1}$. A higher molecular weight of displacer was needed to overcome the adsorption isotope effect, which at the segmental level favours adsorption of the deuterio sample.

Quantitative analysis of how this trapped fraction changed according to the displacement driving force (this quantity was varied by changing the displacer molecular weight or the solution concentration) showed a U-shaped distribution of sticking states [10]. A quantitative histogram of the distribution of adsorbed substates is plotted in figure 14, for the case of $9 \mathrm{~h}$ aging of the initially adsorbed chains before the attempted displacement. The units of the abscissa are a measure of activation energy for displacement (arbitrary units). This relative energy scale is denoted by $\Delta E$.

The peak at small $\Delta E$ reflects the fraction of polymers that were displaced at even the smallest displacer concentrations studied. The histogram is U-shaped, however, and shows the partitioning between tight and loose binding expected from the discussion presented in the previous sections. With increasing equilibration time $(2$ and $9 \mathrm{~h})$ the conformational distribution broadened in the direction of greater uniformity, but remained U-shaped.

\section{Discussion}

We have reported theoretical and experimental analysis of the conformational inhomogeneity which characterizes the non-equilibrium adsorption of polymers from solution. For PMMA, the frequency distribution of the bound fraction of hydrogen bonds along the chain was non-uniform. A relatively large fraction of chains with a high and low bound fraction was obtained, and the distribution was flat for intermediate bound fractions. This distribution remained stale in time for the adsorbed PMMA layers. In contrast, the adsorption of the more weakly surface interacting polymer, PS, exhibited an inhomogeneous layer structure which gradually evolved over periods of hours. Apparently, even these weakly adsorbing chains adsorbed faster than the surface structure could equilibrate, giving rise to an inhomogeneous 


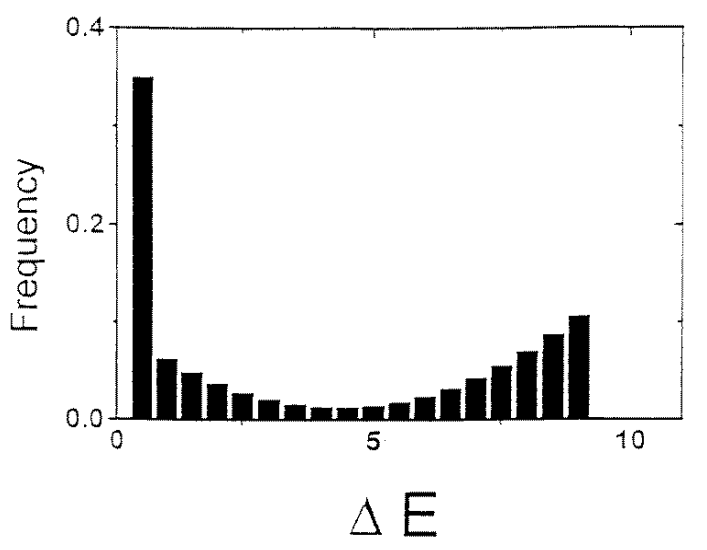

Figure 14. A histogram showing the proposed distribution of conformational heterogeneity in the adsorbed PS layers after an equilibration time of $9 \mathrm{~h} \mathrm{[10].} \mathrm{At} \mathrm{the} \mathrm{smallest} \mathrm{solution}$ concentration (the lowest activation energy for desorption, $\Delta E$ ), the fraction desorbed is largest. The units of $\Delta E$ are arbitrary.

layer structure over rather appreciable time scales. This inhomogeneity was reflected in desorption measurements which were sensitive to this variation in chain conformation structure.

A simple kinetic model was introduced to model the basic features of non-equilibrium polymer adsorption. We assumed that the polymer deposition occurs as an RSA ('parking') of disclike regions on the surface where the size of the discs must be adjusted to fit the available uncovered space where the disc centre lands. This simple model, with a constraint on the upper and lower size of the depositing discs, leads to a distribution of disc areas that is quite similar to the bound fraction distribution found experimentally for non-equilibrium polymer adsorption.

The observation of inhomogeneity in adsorbed polymer layers has implications for understanding desorption processes associated with these films and potentially has practical applications in cases where the tenacity of surface-chain attachment is important (e.g. adhesion or emulsification agents). We can also expect the properties of adsorbed polymer films to evolve slowly even in the case of weakly adsorbing polymers, as for PS in the present study, leading to slow evolution of the above properties over very long time scales.

From a scientific standpoint the polymer adsorption problem provides a model system for understanding the causes and consequences of the geometrical inhomogeneity that characterizes polymer solutions and other complex fluids such as supercooled glass-forming liquids. The surface adsorption problem shows that a distribution of geometrical structure can develop in a chemically homogeneous material and that this structural distribution can lead to properties unique to these materials. When one considers that this structure can be broken down by mechanical forces and affected by the sample history, it is evident that a rich range of material properties becomes possible.

This view of the properties of polymer films and polymeric materials as a whole differs significantly from many traditional models, which assume that structure is dictated by thermodynamic equilibrium or which make the dynamic mean-field theory assumption of a homogeneous molecular environment in polymer materials [11]. An understanding of this kind of structural inhomogeneity is basic to understanding the properties of these complex materials. 


\section{Outlook}

The common occurrence of non-equilibrium dynamics of polymers at surfaces has recently become widely appreciated [27-36] but its conceptual understanding has remained unsatisfactory. Can useful generalizations be made regarding what determines the distribution of heterogeneity given the intrinsically history-dependent nature of these materials?

In this study we show that one essential point is a decoupling of time scales. If chains adsorb from a dilute concentration in solution, they adsorb as discrete chains, more rapidly than the additional time required to achieve conformational equilibration at the surface [2736]. The order of arrival at the surface is then at the heart of the resulting surface structure; chains that arrive first adsorb most tightly, and occupy a disproportionately large number of potential surface adsorption sites, causing the adsorbed chain to be flattened and difficult to extract from the surface. Since the number of segments involved in binding a chain to the surface varies between those chains that were loosely and tightly bound, different fractions of the total layer should be susceptible to different desorption driving forces. This expectation was observed experimentally.

It is worth emphasizing a key feature of this model: chains adapt to the available surface area before the deposition of additional chains. Thus, paradoxically, time need not be considered explicitly when modelling the growth of the adsorbed layer. When modelling adsorption from dilute solution (as in the present experiments), this minimalist assumption seems justified. Introduction of a time variable to describe the adaptation process might be needed for treating adsorption from concentrated solution or the melt state.

\section{A speculative perspective}

It is tempting to extend the argument in analogous fashion to other areas of everyday experience. What determines the ownership of laboratory space in a research organization? Usually one finds that those researchers whose tenure is longest occupy the largest space. What determines the size of industrial firms and of personal fortunes when dealing with a new consumer market? This inhomogeneous distribution is also well known; one thinks, in the United States, of the software entrepreneurs of the 1990s and of the great robber barons of the 1890s. Everyday experience tells us that those to enter the market first acquire the largest wealth, leaving smaller and smaller residual space for latecomers to occupy. Furthermore, there is also a lower cut-off limit, analogous to the lower cut-off limit in the ARSA polymer adsorption problem, set by the minimum economies of scale required to compete at all. This type of model also gives insight into the tendency for the development of power-law distributions for competing entities when the disparities between the sizes of the largest and smallest species can be large.

There are a number of specific situations, beyond the materials science applications of bubble and droplet growth [37] and non-equilibrium polymer adsorption, where it appears plausible to understand the occurrence of hierarchical structures as arising from the adaptive filling of space with excluded volume interactions preventing overlap of the covering species. The dramatic increase of biodiversity during the pre-Cambrian geological period [38] has been interpreted as arising from the first filling of an ecological landscape [39]. In this scenario, existing species exclude the possible existence of other species. A simple geometrical model of the exclusion of lifeforms according to size (which have a high capacity to adaptively fill the space of possible lifeforms) would then lead us by similar arguments to expect a power-law distribution of lifeform density with lifeform size (mass 
or linear dimension [40]). This type of size distribution is commonly observed for lifeforms as a whole [41,42] and even for size distribution of particular lifeforms (e.g. mammals $[40,43])$. Indeed, the ARSA model seems to give insight into the robust occurrence of hierarchical structure in ecosystems [44].

A similar phenomenon may be at work in the growth of large cities through the accretion of townships which cover the space available for city growth. The frequency distribution of these townships within representative large cities (e.g. Berlin and London) exhibits a powerlaw distribution of radius much as expected from the ARSA model $[45,46]$. In the field of economics, this type of model might also have relevance in understanding the power-law distributions that are observed in household income distributions [47], where the space of economic opportunity becomes filled hierarchically because of the wide range of human adaptability and mutual interference in the pursuit of income.

The structural organization can be quite different when the capacity for adaptation is restricted, however. If no adaptation occurs we have uniformity of coverage, but jamming eventually occurs so that significant part of the space remains uncovered (see figure 2). The restriction to an intermediate range of adaptability leads to the interesting situation that near-full coverage occurs, but the size distribution of the covering species can have a peculiar property: the average of the size distribution can be least probable owing to the development of a 'bimodal' or 'U-shaped' frequency distribution.

From this point of view we can whimsically understand, by rational extension, the origin of bimodal distributions of various kinds — of size, wealth, and market share - in the larger natural (including biological) and technological worlds.

Disclaimer statement The reference to commercial equipment does not imply its endorsement by NIST. According to ISO 31-8, the term 'molecular weight' has been replaced by 'relative molecular mass' with the symbol $M_{r}$. The older conventional notation is used in the present work.

\section{Acknowledgments}

We are indebted to S A Sukhisvili (University of Illinois) for many discussions. This work was supported by the Exxon Corporation and by taxpayers of the United States through an NSF Fellowship (to HMS) and the National Science Foundation (Polymers Program).

\section{References}

[1] Stromberg R R, Grant W H and Passaglia E 1964 J. Res. NBS A 68391

[2] Grant W H, Smith L E and Stromberg R R 1975 Faraday Discuss. Chem. Soc. 59209

[3] Pefferkorn E, Haouam A and Varoqui R 1988 Macromolecules 212111

[4] Pefferkorn E, Haouam A and Varoqui R 1989 Macromolecules 222677

[5] Haouam A and Pefferkorn E 1988/89 Colloids Surf. 34371

[6] Zajac M and Chakrabarti A 1996 J. Chem. Phys. 1042418

[7] Schneider H M, Frantz P and Granick S 1996 Langmuir 12994

[8] Enriquez E, Schneider H M and Granick S 1995 J. Polym. Sci., Polym. Phys. Edn. 332429

[9] Frantz P and Granick S 1995 Macromolecules 286915

[10] Schneider H M and Granick S submitted

[11] Fleer G J, Cohen Stuart M A, Scheutjens J M H M, Cosgrove T and Vincent B 1993 Polymers at Interfaces (London: Chapman and Hall)

[12] Schneider H M, Granick S and Smith S 1994 Macromolecules 274714

[13] Evans J W 1993 Rev. Mod. Phys. 651281

[14] van Tassel P R, Viot P, Tarjus G and Talbot J 1994 J. Chem. Phys. 1017064

[15] Ramsden J J 1994 Phys. Rev. Lett. 71295 
[16] Wang J-S and Pandey R B 1996 Phys. Rev. Lett. 771773

[17] Huber G 1990 Correlations and Connectivity: Geometrical Aspects of Physics, Chemistry and Biology ed H E Stanley and N Ostrowky (Dordrecht: Kluwer)

[18] Brilliantov N V et al 1994 J. Phys. A: Math. Gen. 27 L381

[19] Brilliantov N V, Andrienko Yu A, Prapivsky P L and Kurths J 1996 Phys. Rev. Lett. 764058

[20] van Tassel P R, Talbot J, Tarjus G and Viot P 1996 Phys. Rev. E 53785

[21] Karim A et al 1995 J. Physique II 51441

[22] Manna S S and Hermann H J 1991 J. Phys. A: Math. Gen. 24 L481

[23] Huber G et al 1992 Phys. Rev. Lett. 692380

[24] Auroy P and Auvray L 1996 Macromolecules 29337

[25] Frantz P and Granick S 1992 Langmuir 81176

[26] Frantz P and Granick S 1995 Macromolecules 286915

[27] Johnson H E and Granick S 1990 Macromolecules 233367

[28] Johnson H E and Granick S 1992 Science 255966

[29] Douglas J F, Johnson H E and Granick S 1993 Science 2622010

[30] Frantz P and Granick S 1991 Phys. Rev. Lett. 66899

[31] Frantz P and Granick S 1995 Macromolecules 286915

[32] Barford W and Ball R C 1987 J. Chem. Soc. Faraday Trans. I 832515

[33] Chakraborty A K, Shaffer J S and Adriani P M 1991 Macromolecules 245226

[34] Konstadinidis K, Prager S and Tirrell M 1992 J. Chem. Phys. 977777

[35] Granick S 1993 Physics of Polymer Surfaces and Interfaces ed I Sanchez (New York: Manning)

[36] Schneider H M and Granick S 1992 Macromolecules 255054

[37] Aste T 1996 Phys. Rev. E 532571

[38] Gould S J 1989 Wonderful Life: The Burgess Shale and the Nature of History (New York: Norton)

[39] Erwin D H, Valentine J W and Jepkoski J J Jr 1987 Evolution 411177

[40] Schmidt-Nielson K 1984 Scaling: Why is Animal Size So Important? (New York: Cambridge University Press)

[41] Cotgreave P 1994 Sci. Prog. 4457

[42] Damuth J 1987 J. Linnean Soc. 31193

[43] Damuth J Nature 290699

[44] Vrba E S and Eldredge N 1984 Paleobiology 10146

[45] Batty L M and Langley P 1994 Fractal Cities (San Diego: Academic Press)

[46] Makse H A, Havlin S and Stanley H E Nature 377608

[47] Montroll E W and Schlesinger M F 1983 J. Stat. Phys. 32209 